\title{
BIOPELÍCULA: UN MECANISMO DE SUPERVIVENCIA DE Helicobacter pylori
}

\section{BIOFILM: A SURVIVAL MECHANISM OF Helicobacter pylori}

\begin{abstract}
Martín Alonso Bayona-Rojas ${ }^{1^{*}}$, Andrés Julián Gutiérrez-Escobar ${ }^{2^{*}}$
${ }^{1}$ Bacteriólogo, Esp., M.Sc.; ${ }^{2}$ Lic. Biología, M.Sc. Docentes Facultad de Medicina, Grupo de Investigaciones Biomédicas y de Genética Aplicada (GIBGA), Universidad de Ciencias Aplicadas y Ambientales U.D.C.A. Calle 222 No. 55-37, Bogotá, D.C, Colombia. Correo electrónico respectivo: mabayona@udca.edu.co; andresgutierrez@colombia.com, *ambos autores realizaron la misma contribución.
\end{abstract}

Rev. U.D.C.A Act. \& Div. Cient. 16(2): 335-342, Julio-Diciembre, 2013

\section{RESUMEN}

Helicobacter pylori es un patógeno que coloniza el estómago humano, el cual, se ha asociado a múltiples enfermedades gastroduodenales. Dentro de su metabolismo produce una biopelícula, que es un complejo exopolisacarido, que le permite a la bacteria sobrevivir en ambientes desfavorables y ser resistente a la acción de los antibióticos, debido a que previene la penetración completa de estos compuestos. La presente revisión estuvo orientada a realizar una actualización sobre la importancia del biopolímero para la supervivencia de la bacteria y mostrar su trascendencia para la salud pública.

Palabras clave: Microbiología, bacteria, úlcera péptica, resistencia microbiana, cultivo microbiano.

\section{SUMMARY}

Helicobacter pylori is a pathogen which colonizes the human stomach and is associated with multiple gastrointestinal diseases. Metabolism occurs within a biofilm which is a complex exopolysaccharide that allows the bacterium to survive in hostile environments and be resistant to the action of antibiotics, since it prevents complete penetration of these compounds. The present review was designed to perform an update on the importance of the biopolymer regarding the survival of the bacteria and show its public health significance.

Key words: Microbiology, bacterium, peptic ulcer, microbial resistance, microbial culture.

\section{INTRODUCCIÓN}

Helicobacter pylori infecta entre el 50 y el $75 \%$ de la pobla- ción mundial. La infección, se asocia al desarrollo de úlcera péptica y participa en la cadena etiológica multicausal, para el desarrollo de cáncer gástrico. En Colombia, uno de los principales motivos de consulta en los servicios médicos y hospitalarios corresponden a sintomatología de enfermedad ácido péptica (Bessa et al. 2012; Gisbert, 2011; Romo \& Coria, 2010; Vale \& Vítor, 2010; Torres \& Backert, 2008; Fuccio et al. 2007; Parkin, 2006; Sierra, 2002).

Los reservorios del microorganismo son el hombre y los animales domésticos, como el perro y el gato y presenta diversos mecanismos de transmisión, por mencionar el agua y los alimentos contaminados, transmisión oro-fecal e insectos, como la mosca doméstica (Ford \& Axon, 2010). La infección no es exclusiva para un rango de edad específico y afecta tanto población infantil (principalmente, en países con condiciones sanitarias pobres) como adulta. Los patrones de infección varían de acuerdo a la zona geográfica y se ha identificado que se asocia con hábitos y con costumbres alimentarias, que influyen en la aparición de sintomatología (Vale \& Vítor, 2010; Flatland, 2002; Gómez \& Orozco, 2006; Kivi \& Tindberg, 2006).

Desde el punto de vista microbiológico, H. pylori es pleomórfico, Gram negativo y habita tanto en el epitelio del estómago como del intestino de humanos y de los animales. Una vez coloniza la mucosa gástrica es difícil erradicarla, a menos que se emplee tratamiento antibiótico. Helicobacter presenta las siguientes características: es microaerófilo, posee una membrana externa y, en promedio, seis flagelos polares estan protegidos por una estructura lipídica; asimismo, es ureasa, catalasa y citocromo-oxidasa positivo, no produce hidrólisis del hipurato ni ácido sulfhídrico. Entre los factores de virulencia, se encuentra la ureasa, que transforma la urea en amoniaco y agua, alcalinizando así el medio ácido cir- 
cundante; también se encuentran lipasas, adhesinas, factor activador plaquetario, la proteína Cag A, Pic B (que induce a citocinas) y la proteína vacuolizante Vac A. (Naranjo et al. 2012a; 2012b; Blanchard \& Nedrud, 2012; Terebiznik et al. 2006; Marshall, 2002).

El cultivo se realiza, principalmente, a partir de biopsias de mucosa gástrica; sin embargo, se logra también empleando muestras extra-gástricas, como placa dental, heces fecales, saliva y muestras obtenidas, a partir de esófago. Los crecimientos típicos, se obtienen entre 4 a 7 días, en condiciones de: $5-10 \%, \mathrm{O}_{2} ; 5-10 \%, \mathrm{CO}_{2} ; 80-90 \%, \mathrm{~N}_{2}$; humedad de $95 \%$ y temperatura de 35 a $37^{\circ} \mathrm{C}$. Para su pleno crecimiento, se emplean medios de cultivo complejos, suplementado con sangre, con vitaminas y con antibióticos (Blanchard \& Nedrud, 2012; Siavoshi et al. 2012; Abrante et al. 2012; Joo et al. 2010; Stevenson et al. 2003; Cellini et al. 2010; Bayona, 2013).

Genéticamente, $H$. pylori es muy diverso, con una tasa de intercambio de material genético alta y con ventajas evolutivas diferenciales entre cepas de la misma especie, tanto para marcadores de virulencia como para adhesinas (Baltrus et al. 2008). En nuestra experiencia, esta diversidad se puede apreciar en el crecimiento diferencial de las cepas en medios de cultivo diferentes (agar Brucella, Campylobacter y Tripticasa de soya); inclusive, cepas aisladas, a partir de biopsias de un mismo paciente, presentan cinéticas de crecimiento diferenciales. Además, análisis evolutivos realizados por nuestro grupo ponen de manifiesto procesos de diferenciación genética entre adhesinas pertenecientes a cepas del este y el oeste, que presentan selección positiva operante (Gutiérrez-Escobar, 2013). Finalmente, mencionar que hasta las características macroscópicas e, inclusive, la coloración de Gram, son diferenciales (Bayona, 2013).

Andersen \& Rasmussen (2009), mediante estudios de microscopía electrónica, observaron formas espirales, formas cocoides y formas degenerativas. Las formas espirales son viables, cultivables, virulentas y pueden colonizar los animales de experimentación. Las formas cocoides también pueden ser viables, pero no cultivables, son menos virulentas y menos propensas a colonizar e inducir inflamación en animales de experimentación. Finalmente, las formas degenerativas no pueden ser cultivadas y la membrana celular se ha desintegrado, pero el material genético puede ser demostrado por la técnica de la reacción en cadena de la polimerasa (PCR), especialmente, en los suministros de agua.

Otro aspecto importante es el enfoque del tratamiento actual que busca eliminar la bacteria, acompañado de una terapia complementaria, que mejora el efecto de los antibióticos, por diferentes mecanismos. A nivel mundial, la terapia estándar ha perdido eficacia, todo debido a la resistencia que ha veni- do desarrollando este microorganismo en los últimos años. En muchos casos, se ignora la efectividad de los esquemas de tratamiento prescritos, ya que no se realizan pruebas para verificar su eliminación (cultivo), pruebas de susceptibilidad y estudios farmacogenómicos (Mégraud \& Corti, 2009; Otero et al. 2009).

Un tema poco mencionado y que consideramos importante desde diversos puntos de vista es el hecho que la bacteria forme una biopelícula; sin embargo, poco es el conocimiento concentrado en nuestro idioma sobre referente a este tema. En el presente artículo, se realizó una revisión de aspectos microbiológicos asociados con la biopelícula producida por H. pylori. Para la búsqueda bibliográfica, se exploraron las siguientes bases de datos: Medline, Proquest, Embase, Jstore,Pubmed,Hinari, Springer, Nature, Science online y Oxford Journal y en revistas particulares como Plos, Nas e Imbiomed. Se combinaron los términos: biofilms and Helicobacter, Helicobacter pylori survival, Helicobacter and Maintenance, Supplements for Helicobacter, Laboratory Maintenance, Susceptibility to antibiotics.

Los métodos de recuperación en caldo y en medios sólidos recomendados por los autores han sido evaluados con cepas de referencia. Cabe resaltar que a cada una de las cepas que hemos obtenido se les ha realizado un control bioquímico, caracterizado por pruebas convencionales (Gram, oxidasa, catalasa y ureasa) y un control molecular basado en la genotipificación de la subunidad $16 \mathrm{~S}$ del gen ribosomal, teniendo en cuenta que estas cepas presentan altísima variabilidad genética. Destacamos, también, que a partir de los cultivos en medio líquidos y sólidos, se presenta como característica importante, la presencia de una biopelícula, cuya secreción es aparentemente diferencial entre las cepas aisladas hasta el momento. Por otra parte, hemos observado las diferentes morfologías macroscópicas de las colonias, lo cual, nos describe su amplia variabilidad genética (Figura 1).

\section{BIOPELÍCULAS}

Algunas bacterias forman conglomerados o comunidades microbianas cuando crecen en un nicho ecológico distinto a su hospedero. Estas comunidades microbianas, se embeben dentro de una matriz orgánica polimérica autoproducida y adherida a una superficie viva (biofilm de mucosa) o inerte, que pueden ser mono o polimicrobianas (Davey \& O'toole, 2000; Kraigsley et al. 2002; Percival \& Thomas, 2009; Coticchia et al. 2006; Costerton et al. 1999; Donlan, 2002; Yonezawa et al. 2010; Binkowska et al. 2013). Las biopelículas son sólidas y presentan canales por donde fluyen el agua y los nutrientes a las zonas más profundas (Yonezawa et al. 2010; Ceyhan, 2010; Di Campli et al. 2010; Makipour \& Friedenberg, 2011). Los primeros análisis de la producción de biofilm por parte de bacterias, como H. pylori, se llevaron 


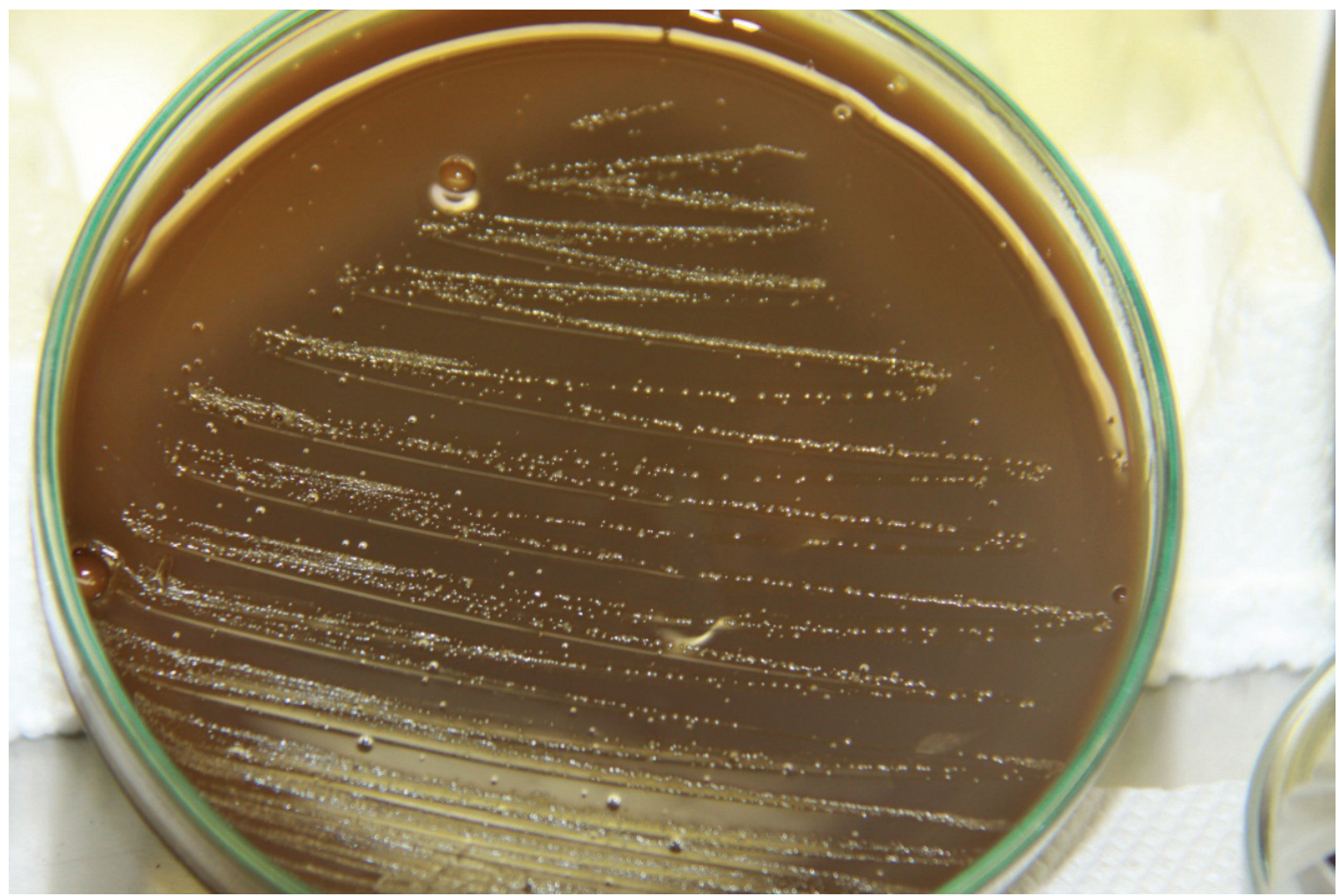

Figura 1. Características macroscópicas de cepas de Helicobacter pylori en medio de cultivo agar Chocolate.

a cabo en Perú, mostrando un fuerte potencial de infección, a partir de muestras de agua potable (Hulten et al. 1996). Los microorganismos que están alojados en la biopelícula tienen diferente metabolismo, crecimiento, disponibilidad de nutrientes, presión osmótica y densidad poblacional microbiana y se comunican por medio de señales químicas. Las células microbianas viven en estrecha proximidad, que les permiten intercambiar material genético, presente en los plásmidos (Binkowska et al. 2013).

Las biopelículas están presentes en todas partes y afectan todos los aspectos de nuestra vida. A nivel clínico, se asocian con neumonía nosocomial, infecciones del tracto urinario (catéter), infecciones supurativas, infecciones del sistema nervioso central, sepsis bacteriana y juegan un papel importante, asociados con fibrosis quística y enfermedad del legionario (Brelles Mariño, 2012; Binkowskawa et al. 2013). Representan una estrategia adaptativa de las bacterias que es ventajosa porque: 1) Protege los microorganismos de agentes adversos, como son desinfectantes y antibióticos y otros agentes ambientales estresantes; 2) Incrementa la disponibilidad de nutrientes, ya que los concentra; 3) Evita la deshidratación y, 4) Posibilita la transferencia de material genético (Costerton et al. 1999; Donlan, 2002). Varios mecanismos están probablemente relacionados con la resistencia antimicobiana: 1) Lenta penetración del agente antimi- crobiano en la biopelícula; 2) Cambio en el microambiente químico dentro de la biopelícula, lo cual, conduce a zonas de crecimiento lento o nulo; 3) Respuesta de estrés de adaptación y, 4) Presencia de una pequeña población de células de extrema resistencia (Huynh et al. 2004).

Grande et al. (2011), al estudiar y caracterizar el ADN extracelular (EDNA) en las biopelículas de $H$. pylori, determinaron que el EDNA no es el componente principal de la matriz y que, como tal, puede estar involucrado en otros procesos que están orientados a la recombinación, a través de la transformación, contribuyendo así a la variabilidad genética de la bacteria.

Las biopelículas también juegan un papel importante, a nivel de salud pública, debido a su papel en enfermedades infecciosas, relacionadas con dispositivos. De acuerdo con la estimación dada por el centro para el control de enfermedades (Atlanta, GA, EE.UU), se estima que 65\% de las enfermedades humanas causadas por infecciones bacterianas están involucradas con biopelículas (Jain et al. 2007).

Estructuralmente, las biopelículas están constituidas por tres componentes: a) masa microbiana, b) espacios intercelulares o canales y c) mMatriz extracelular que rodea el complejo y está compuesta de exopolisacaridos, proteínas, 
ácidos nucleicos y otras sustancias (Costerton et al. 1987). La formación de la biopelícula está regulada por un sistema de señales dependiente de la acumulación de un autoinductor. En bacterias Gram negativas, como H. pylori, el principal autoinductor es la acil-homoserina lactona (Donlan, 2002; Lasa et al. 2009). La formación de la biopelícula conlleva un proceso sistemático de cinco fases: 1) Adsorción reversible de la bacteria a la superficie; 2) Unión irreversible a la superficie; 3) Fase inicial de maduración con crecimiento y desarrollo microbiano; 4) Producción del exopolímero y, 5) Desarrollo final de la colonia con dispersión de células colonizadoras (Kumar \& Anand, 1998; Cole et al. 2004).

Un medio común para la formación de biopelículas es el agua, frente a lo cual, los estudios epidemiológicos sugieren que el agua del medio ambiente es un factor de riesgo para su infección, en comparación con el agua del grifo (Adams et al. 2003; Watson et al. 2004; Bellack et al. 2006). Muchas veces, el tratamiento con antimicrobianos de comunidades de bacterias patógenas es inefectivo (Patel, 2005).

Las biopelículas pueden presentar diferentes formas dependientes del ambiente, sea natural, clínico o industrial (Davey \& O' Toole, 2000; Kraigsley et al. 2002; Cammarota et al. 2010). Dentro de esos ambientes encontramos: piel, tracto intestinal, alrededor de raíces vegetales, en las tuberías, en la placa dental o en instrumentos implantados, como catéteres, marcapasos y prótesis (Donlan, 2002; Serra, 2003); sin embargo, no parece participar en la formación de biopelícula en la cavidad oral, a pesar que la bacteria se puede detectar (Andersen \& Rasmussen, 2009).

Bessa et al. (2012) evaluaron, a nivel in vitro, el comportamiento fisiológico de biopelículas de $H$. pylori y emplearon cuatro medios de cultivo líquido: caldo Brucella, infusión de cerebro corazón, caldo Ham F-12 adicionado de $2 \%$ de suero fetal de ternero y Ham sin suero. La formación de las biopelículas fue significativamente dependiente de los medios empleados, recomendando los medios Ham F-12. Posteriormente, Bessa et al. (2012), con el fin de obtener estudios fisiológicos más precisos, optimizaron condiciones de cultivo para $H$. pylori, los cuales, incluyeron al medio Ham F-12 suplementado con suero fetal bovino al $5 \%$. La estrategia del diseño experimental mostró resultados óptimos, en valores de $\mathrm{pH}(8,0)$, velocidad de agitación, (130rpm), valor de tasa de crecimiento específico de 0,164 h-1, correspondiente a una concentración máxima aproximada de 1,5x $108 \mathrm{UFC} / \mathrm{ml}$, después de 8 horas. En contraste a lo anterior, Yonezawa et al. (2010), en su estudio de formación de biopelícula de $H$. pylori, obtuvo un óptimo crecimiento en caldo Brucella suplementado con suero de ternera fetal al $7 \%$, encontrando que no hubo diferencias significativas en la autoagregación, la motilidad y la hidrofobicidad de las diferentes cepas evaluadas.
Un trabajo que empleó caldo Brucella adicionado de suero de caballo al $10 \%$ fue evaluado por Joo et al. (2010), quienes introdujeron una nueva técnica en capa fina líquida, empleando como soporte, caja de Petri de $90 \mathrm{~mm}$. Esta metodología representa una alternativa versátil para la investigación del microorganismo. Una cepa de $H$. pylori NCTC 11637 produjo una biopelícula, bajo condiciones in vitro, en un medio con un alto contenido de carbono (caldo Brucella), suplementado con $3 \mathrm{~g}$ de glucosa. En nuestra experiencia, se ha dado un resultado similar, al observar presencia del exopolisacarido, a las 18 horas de iniciado el cultivo.

Carron et al. (2006) demostraron, mediante microscopia electrónica de barrido (primer registro fotográfico), la existencia de una biopelícula de $H$. pylori en mucosa gástrica humana. Los pacientes evaluados, se sometieron a una endoscopia, tomándose tres muestras por cada uno; paralelamente, se realizaron pruebas confirmatorias de ureasa.

Cammarota et al. (2012) demostraron, mediante un escaneo por medio de la microscopia electrónica, a partir de biopsias gástricas obtenidas de pacientes infectados por $H$. pylori, la formación de biopelículas. Estos biopolímeros permiten que el microorganismo permanezca latente en este medio gástrico y asimismo, sea resistente a la acción de los antibióticos. Mediante el empleo de N-acetilcisteína, antes de iniciar el tratamiento con antibióticos, permitió erradicar la biopelícula, a nivel gástrico y, de esta manera, poder eliminar posteriormente la bacteria. Asimismo, Makipour \& Friedenberg (2011) y Huynh et al. (2004) mostraron el papel que presenta este compuesto en la degradación de la biopelícula.

Souto \& Vieira (2008), al evaluar la prevalencia de H. pylori por PCR en biofilm subgingival y de saliva de 169 pacientes con periodontitis crónica, encontraron una prevalencia de un $20 \%$. Se observó una mayor prevalencia en muestras de biopelícula subgingival $(33,3 \%)$, en comparación con muestras de saliva (20\%), permitiendo establecer que dicho microorganismo se detecta con frecuencia en la microbiota oral de sujetos con periodontitis. Por otra parte, el trabajo llevado a cabo por Ghosh \& Bodhankar (2012), quienes evaluaron 1.500 muestras de saliva de pacientes asintomáticos para H. pylori por PCR, hallaron una prevalencia en hombres de $75,96 \%$ y en mujeres de $88,10 \%$. El papel de la cavidad oral en la colonización por $H$. pylori es considerada como transitoria e independiente del estado del paciente, de tal forma que el tiempo de supervivencia debe ser estudiado con el fin de aumentar su conocimiento sobre la vía de transmisión de este patógeno (Al-Ahmad et al. 2012).

Se ha demostrado que en agua, las biopelículas protegen a los microorganismos de condiciones adversas (Percival \& Thomas, 2009; Stewart \& Costerton, 2001). Técnicas, 
como PCR y la prueba de fluorescencia de hibridación in situ (FISH), se han empleado con éxito para identificar con satisfacción la presencia de H. pylori en agua (Giao et al. 2008).

Cuando se habla de la presencia de $H$. pylori en agua, se define como un microorganismo que tiene la capacidad de entrar en estado viable, pero no cultivable, sobre todo en casos de condiciones desfavorables. Frente a esta problemática, Linke et al. (2010) evaluaron la prueba de PCR en tiempo real, para lo cual, las biopelículas con el microorganismo se generaron en un modelo con agua potable sobre un tubo con silicona. Dentro de los resultados obtenidos evidenciaron que la secuencia de ADN de la sonda y los cebadores no mostró homología cruzada con otras bacterias, siendo posible detectar diez unidades genómicas, representando, por lo tanto, una nueva herramienta de análisis para este tipo de microorganismo.

\section{CONCLUSIONES}

A pesar de las importantes implicaciones microbiológicas y fisiológicas que presenta la formación de las biopelículas no es mucho lo que se conoce sobre su producción, regulación y papel en la resistencia a los antibióticos. El hecho de poder conocer e identificar elementos genéticos y factores ambientales asociados con la formación de biopelículas permitirá establecer estrategias efectivas para su control. El objetivo de esta revisión fue, precisamente, traer a la retina de la comunidad científica nacional e internacional, de habla hispana, este tema, para abrir posibilidades investigativas en nuestras regiones, ya que poco se conoce sobre la formación de biopelículas y sus implicaciones clínicas, por parte de cepas nativas para la región latinoamericana.

Conflicto de intereses: El artículo fue preparado y revisado con la participación de todos los autores, quienes declaramos que no existe ningún conflicto de intereses, que ponga en riesgo la validez de los resultados presentados.

\section{BIBLIOGRAFÍA}

1. ABRANTE, L.; REYES, N.; GARCÍA, M.; SUÁREZ, P.; ROMERO, R.; MICHELANGELI, F.; CONTRERAS, $M$. 2012. Diagnóstico de la infección por Helicobacter pylori por PCR en jugo gástrico y biopsias gastroesofágicas de pacientes dispépticos. Invest. Clin. 53(2): 168-177.

2. ADAMS, B.; BATES, T.; OLIVER, J. 2003. Survival of Helicobacter pylori in a natural freshwater environment. Appl. Environ. Microbiol. 69(12):74627466.
3. AL-AHMAD, A.; KURSCHER, A.; AWECKESSER, S.; WITTMER, A.; RAUBERGER, H.; JAKOB, T.; HELLWIG, E.; KIST, M.; WAIDNER, B. 2012. Is Helicobacter pylori resident or transient in the human oral cavity? J. Med. Microbiol. 61(8):11461152.

4. ANDERSEN, L.; RASMUSSEN, L. 2009. Helicobacter pylori-coccoid forms and biofilm formation. FEMS Immunol. Med. Microbiol.56(2):112-115.

5. BALTRUS, D.; GUILLEMIN, K.; PHILLIPS, P. 2008. Natural transformation increases the rate of adaptation in the human pathogen Helicobacter pylori. Evolution. 62(1):39-49.

6. BAYONA, M. 2013. Condiciones microbiológicas para el cultivo de Helicobacter pylori. Rev. Col. Gastroent. 28 (2):94-99.

7. BELLACK, N.; KOEHOOM, M.; MACNAB, Y.; MORSHED, M. 2006. A conceptual model of waters role as a reservoir in Helicobacter pylori transmission: a review of the evidence. Epidemiol. Infect. 134(3):439449.

8. BESSA, L.; CORREIA, L.; CELLINI, L.; AZEVEDO, N.; ROCHA, I. 2012. Optimization of culture conditions to improve Helicobacter pylori growth in Ham's F-12 medium by response surface methodology. Int J. Pharmacol. Immunopath. 25(4):901-909.

9. BINKOWSKA, A.; BIERNAT, M.; DUS, I.; GOSCINIAK, G. 2013. The role of biofilm formation in pathogenesis of Helicobacter pylori infections. Prz Gastroenterol. 8(1):27-30.

10. BLANCHARD, T.; NEDRUD, J. 2012. Laboratory maintenance of Helicobacter species. Current Protocols in Microbiology. Suppl.24, 23p.

11. BRELLES MARIÑO, G. 2012. Challenges in biofilm inactivation: the use of cold plasma as a new approach. J. Bioproces. Biotechnig. 2(14):1-4.

12. CAMMAROTA, G.; SANGUINETTI, M.; GALLO, A.; POSTERARO, B. 2012. Biofilm formation by Helicobacter pylori as a target for eradication of resistant infection. Aliment. Pharmacol. Ther. 36(3):222-230.

13. CAMMAROTA, G.; BRANCA, G.; ARDITO, F.; SANGUINETTI, M.; IANIRO, G.; CIANCI, R.; 
TORELLI, R.; MASALA, G.; GASBARRINI, A.; FADDA, G.; LANDOLFI, R.; GASBARRINI, G. 2010. Biofilm demolition and antibiotic treatment to eradicate resistant Helicobacter pylori: A clinical trial. Clin. Gastroent. Hepatol. 8:817-820.

14. CARRON, M.A.; TRAN, V.R.; SUGAWA, C.; COTICCHIA, J.M. 2006. Identification of Helicobacter pylori biofilms in human gastric mucosa. J. Gastrointest. Surg.10(5):712-717.

15. CELLINI, L.; GRANDE, R.; ARTESE, L.; MARZIO, L. 2010. Detection of Helicobacter pylori in saliva and esophagus. New Microbiol. 33(4):351-357.

16. CEYHAN, N. 2010. Formation of biofilm in drinking water by a gastric pathogen Helicobacter. J. BIBAD, Biyoloji Bilimleri Arastirma Dergisi. 3(2):115-119.

17. COLE, S.; HARWOOD, J.; LEE, R.; SHE, R.; GUINEY, D. 2004. Characterization of monospecies biofilm formation by Helicobacter pylori. J. Bacteriol. 186(10):3124-3132.

18. COSTERTON, J.W.; CHEN, G.; GERSEY, T.I.; LADD, J.G.; NICKEL, M. 1987. Bacterial biofilms in nature and disease. Ann. Rev. Microbiol. 41:435-64.

19. COSTERTON, J.; STEWART, P.; GREENBERG, E. 1999. Bacterial Biofilms: a common cause of persistent infections. Science. 284:1318-1322.

20. COTICCHIA, J.; CHOICHI, S.; TRAN, V.; GURROLA, J.; KOWALSKI, E.; CARRON, M. 2006. Presence and density of Helicobacter pylori biofilms in human gastric mucosa in patients with peptic ulcer disease. J. Gastrointest. Surg. 10(6):883-889.

21. DAVEY, M.; O' TOOLE, G. 2000. Microbial biofilms: from ecology to molecular genetics. Microbiol. Mol. Biol. Reviews. 64(4):1092-2172.

22. DI CAMPLI, E.; DI BARTOLOMEO, S.; GRANDE, R.; DI GIULIO, M.; CELLINI, L. 2010. Effects of extremely low- frequency of electromagnetic fields on Helicobacter pylori biofilm. Current Microbiol. 60(6):412-418.

23. DONLAN, R. 2002. Biofilms: microbial life on surfaces. Emerg. Infect. Diseas. 8(9):881-890.

24. FLATLAND, B. 2002. Helicobacter infection in humans and animals. Comp. Cont. Educ. Pract. Vet. 24(9):688-698.
25. FORD, A.C.; AXON, A.T. 2010. Epidemiology of Helicobacter pylori infection and public health implications. Helicobacter. 15(Suppl1):1-6.

26. FUCCIO, L.; ZAGARI, R.; MINARDI, M.; BAZZOLI , F. 2007. Systematic review: Helicobacter pylori eradication for the prevention of gastric cancer. Aliment. Pharma .Therap. 25(2):133-141.

27. GHOSH, P.; BODHANKAR, S. 2012. Determination of risk factors and transmission pathways of Helicobacter pylori in asymptomatic subjects in Western India using polymerase chain reaction. Asian Pacific J. Trop. Disease. 2(1):12-17

28. GIAO, M.; AZEVEDO, N.; WILKS, S.; VIEIRA, M.; KEEVIL, C. 2008. Persistence of Helicobacter pylori in heterotrophic drinking-water biofilms. Appl. Environm. Microbiol. 74(19):5898-5904.

29. GISBERT, J. 2011. Helicobacter pylori related diseases; dyspepsia, ulcers and gastric cancer. Gastroenterol. Hepatol. 34(2):15-26.

30. GÓMEZ, L.; OROZCO, S. 2006. Helicobacteriosis canina y felina. Vet. Méx. 37:97-116.

31. GRANDE, R.; DI GIULIO, M.; BESSA, L.; DI CAMPLI, E.; BAFFONI, M.; GUARNIERI, S.; CELLINI, L. 2011. Extracellular DNA in Helicobacter pylori biofilm: a backstairs rumour. J. Appl. Microbiol. 110(2):490498.

32. GUTIÉRREZ-ESCOBAR, A.J. 2013. Análisis evolutivo de la adhesión: evidencia de selección positiva operante sobre el locus AlpAB y el gen horB de Helicobacter pylori. Rev. U.D.C.A Act. \& Div. Cient. 16(1):3-15.

33. HULTEN, K.; HAN, S.; ENROTH, H.; KLEIN, P.; OPEKUN, A.; GILMAN, R.; EVANS, D.; ENGSTRAND, L.; GRAHAM, D.; EL-ZAATARI, F. 1996. Helicobacter pylori in the drinking water in Peru. Gastroenterol.110:1031-1035.

34. HUYNH, H.Q.; COUPER, R.T.; TRAN, C.; MOORE, L.; KELSO, R.; BUTLER, R. 2004. N- Acetilcysteine, a novel treatment for Helicobacter pylori infection. Digest. Dis. Sci. 49 (11-12):1853-1861.

35. JAIN, A.; GUPTA, Y.; AGRAWAL, R.; KHARE, P.; JAIN, S.K. 2007. Biofilms--a microbial life perspective: a critical review. Crit. Rev. Ther. Drug Carrier Syst. 24(5):393-443. 
36. JOO, J.; PARK, K.; SONG, J.; KIM, D.; LEE, K.; KWON, Y.; KIM, J.; KIM, K.; YOUN, H.; KANG, H.; BAIK, S.; LEE, W.; CHO, M.; RHEE, K. 2010. Thin-layer liquid culture technique for the growth of Helicobacter pylori. Helicobacter. 15(4):295-302.

37. KIVI, M.; TINDBERG, Y. 2006. Helicobacter pylori occurrence and transmission: a family affair? Scand. J. Infect.Dis. 38(6-7):407-417.

38. KRAIGSLEY, A.; RONNEY, P.; FINKEL, S. 2002. Dynamics of self-propagating fronts of motile bacteria. Disponible desde internet en: http://carambola.usc. edu/research/biophysics/BacterialFronts.html (con acceso 10/11/2012).

39. KUMAR, C.; ANAND, S. 1998. Significance of microbial biofilms in food industry: a review. Int. J. Food Microbiol. 42:9-27.

40. LASA, I.; DEL POZO, J.; PENADÉS, J. 2009. Biofilms bacterianos e infección. Disponible desde internet en: http:/www.cfnavarra.es/salud/anales/textos/ vol28/n2/colaba.html (con acceso 13/10/2012.

41. LINKE, S.; LENZ, J.; GEMEIN, S.; EXNER, M.; GEBEL, J. 2010. Detection of Helicobacter pylori in biofilms by real-time PCR. Int. J. Hyg. Environm. Health. 213(3):176-182.

42. MAKIPOUR, K.; FRIEDENBERG, F. 2011. The potential Role of $\mathrm{N}$-acetylcysteine for the treatment of Helicobacter pylori. J. Clin. Gastroent. 45(10):841843.

43. MARSHALL, B. 2002. Helicobacter pylori: 20 years on. Clin. Med. 2(2):147-152.

44. MÉGRAUD, F.; CORTI, R. 2009. Resistencia bacteriana del Helicobacter pylori en el mundo en el año 2009. Acta Gastroent. Latinoam. 39(4):282-290.

45. NARANJO, D.; SUÁREZ, M.; BAYONA, M.; GALLEGO, M.; URBINA, M.; ROJAS, D.P. 2012a. Aspectos históricos, epidemiológicos y patológicos de la helicobacteriosis en humanos y en caninos. Rev. Medicina. 34(2):146-161.

46. NARANJO, D.; SUÁREZ, M.; ROJAS, D.P.; BAYONA, M.; GALLEGO, M.; URBINA, M. 2012b. Relación de helicobacteriosis en caninos y sus propietarios en diferentes estratos socioeconómicos en Bogotá D.C. Medicina. 34(3):211-220.
47. OTERO, W.; TRESPALACIOS, A.; OTERO, E. 2009. Helicobacter pylori: Tratamiento actual. Un importante reto en gastroenterología. Rev. Col. Gastroenterol. 24(3):279-292.

48. PARKIN, D. 2006. The global health burden of infectionassociated cancers in the year 2002. Int. J. Cancer. 118(12):3030-3044.

49. PATEL, R. 2005. Biofilm and antimicrobial resistance. Clin. Orthop. Relat. Res. 437:41-47.

50. PERCIVAL, S.; THOMAS, J. 2009. Transmission of Helicobacter pylori and the role of water and biofilms. J. Water Health. 7:469-477.

51. ROMO, C.; CORIA, V.R. 2010. Helicobacter pylori, un modelo de bacteria carcinogénica. Rev. Especial. Médico-Quirúrg. 15(4):242-251.

52. SERRA, P.G. 2003. Estudio de biofilms: formación y consecuencia. Disponible desde internet en: http:// magno.uab.es/epsi/alimentaria/ biofilm.pdf (con acceso 13/10/2012].

53. SIAVOSHI, F.; SANIEE, P.; ATABAKHSH, M.; PEDRAMNIA, S.; TAVAKOLIAN, A.; MIRZAEI, M. 2012. Mucoid Helicobacter pylori isolates with fast growth under microaerobic and aerobic conditions. Helicobacter. $17: 62-67$

54. SIERRA, F. 2002. Helicobacter pylori-Estado actual. Rev. Col. de Cirugía. 17(3):128-130. Disponible desde internet en http://www.encolombia.com/ medicina/cirugia/ciru17302editorial.htm (con acceso 13/10/2012).

55. SOUTO, R.; VIEIRA, A.P. 2008. Detection of Helicobacter pylori by polymerase chain reaction in the subgingival biofilm and saliva of non-dyspeptic periodontal patients. J. Periodontol. 79(1):97-103.

56. STEVENSON, T.; LUCIA, L.; ACUFF, G. 2003. Development of a selective medium for isolation of Helicobacter pylori from cattle and beef samples. Appl. Environ. Microbiol. 66:723-727.

57. STEWART, P.; COSTERTON, J. 2001. Antibiotic resistance of bacteria in Biofilms. Lancet. 358:135-138.

58. TEREBIZNIK, M.R.; VÀSQUEZ, C.L.; TORBICKI, K.; BANKS, D.; WANG, T.; HONG, W.; BLANKE, S.R.; COLOMBO, M.I.; JONES, N.L. 2006. Helicobacter pylori VacA toxin promotes bacterial intracellular 
survival in gastric epithelial cells. Infect. Immun. 74(12):6599-6614.

59. TORRES, J.; BACKERT, S. 2008. Pathogenesis of Helicobacter pylori infection. Helicobacter, 13(1):13-17.

60. VALE, F.; VÍTOR, J. 2010. Transmission pathway of Helicobacter pylori: does food play a role in rural and urban areas? Int. J. Food Microbiol. 138(1-2):112.

61. WATSON, C.L.; OWEN, R.J.; SAID, B.; LAI, S.; LEE, J.V.; SUMAN-LEE, S.; NICHOLS, G. 2004. Detection of Helicobacter pylori by PCR but not culture in water and biofilm samples from drinking water distribution systems in England. J. Appl. Microbiol. 97:690-698.

62. YONEZAWA, H.; OSAKI, T.; KURATA, S.; ZAMAN, C.; HANAWA, T.; KAMIYA, S. 2010. Assessment of in vitro biofilm formation by Helicobacter pylori. J. Gastroent. Hepatol. 25(Suppl 1):S90-94.

Recibido: Abril 15 de 2013

Aceptado: Octubre 7 de 2013

Como citar:

Bayona-Rojas, M.A.; Gutiérrez-Escobar, A.J. 2013. Biopelícula: un mecanismo de supervivencia de Helicobacter pylori. Rev. U.D.C.A Act. \& Div. Cient. 16(2): 335-342. 